October 28, 2018

\title{
The Berry Phase of D0-Branes
}

\author{
Chris Pedder, Julian Sonner and David Tong \\ Department of Applied Mathematics and Theoretical Physics, \\ University of Cambridge, UK
}

\begin{abstract}
We study $S U(2)$ Yang-Mills quantum mechanics with $N=2,4,8$ and 16 supercharges. This describes the non-relativistic dynamics of a pair of D0-branes moving in $d=3,4,6$ and 10 spacetime dimensions respectively. We show that as the D0-branes orbit, states undergo a Berry holonomy described by the four Hopf maps. For the $N=2$ theory, the associated Hopf map is the $\mathbf{Z}_{2}$ Möbius bundle and its effect is to turn the D0-branes into anyons with exchange statistics $e^{i \pi / 2}$. For the $N=4,8$ and 16 theories, the Hopf maps give rise to Berry connections that are familiar to physicists: the $U(1)$ Dirac monopole; the $S U(2)$ Yang monopole; and the $S O(8)$ octonionic monopole.
\end{abstract}




\section{Introduction}

Many years ago, Kugo and Townsend [1] pointed out a relationship between supersymmetric field theories with $N=2,4,8$ and 16 supercharges and the four normed division algebras $\mathbb{K} \cong \mathbb{R}, \mathbb{C}, \mathbb{H}$ and $\mathbb{O}$. The key observation is algebraic. Theories with $N=2,4,8$ and 16 supercharges naturally live in $d=3,4,6$ and 10 spacetime dimensions respectively. The Lorentz Lie algebra in these dimensions is isomorphic to the algebra of $2 \times 2$ Hermitian matrices with vanishing trace and elements in $\mathbb{K}$,

$$
\operatorname{sl}(2 ; \mathbb{K}) \cong \operatorname{so}(d-1,1)
$$

This allows us to express a spinor in $d$ dimensions as a 2-component $\mathbb{K}$-vector, generalizing the well-known result for $d=4$. This construction was elaborated upon in $[2]$.

For theories with $N=2,4$ and 8 supercharges, the relationship to the division algebra $\mathbb{K}$ also manifests itself in more physical and dynamical matters. This includes familiar features of supersymmetric theories, such as the holomorphy of the superpotential and the hyperKähler/quaternionic structure of Calabi-Yau moduli spaces. However, so far the tantalizing idea that an octonionic structure underlies theories with 16 supercharges has not led to major insight about quantum dynamics.

The purpose of this short note is to point out that the isomorphism (11) has a simple, physical consequence in the framework of supersymmetric quantum mechanics. We focus on $S U(2)$ gauged quantum mechanics. The theory with $N$ supercharges can be thought of as the dimensional reduction of the minimal super Yang-Mills theory in $d=3,4,6$ and 10 dimensions, and describes the non-relativistic dynamics of two D0-branes moving in $d-1=N / 2+1$ spatial dimensions. We show that the states of a pair of orbiting D0-branes undergo a holonomy described by the Hopf map associated to the division algebra $\mathbb{R}, \mathbb{C}, \mathbb{H}$ and $\mathbb{O}$.

The four Hopf maps take $S^{N-1} \rightarrow S^{N / 2}$ :

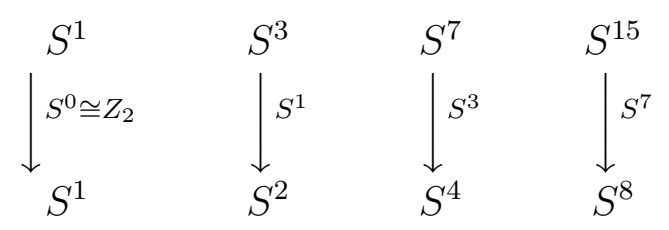

The case of $N=2$ supercharges is special. The map $\mathbf{Z}_{2} \hookrightarrow S^{1} \rightarrow S^{1}$, sometimes known as the zeroth Hopf map, describes the Möbius bundle. We will show in Section 3.1 
that the ground state of the associated quantum mechanics undergoes a discrete $\mathbf{Z}_{2}$ holonomy, changing by a sign as D0-branes orbit. Since a single orbit is equivalent to two exchanges, this implies that the D0-branes in $N=2$ Yang-Mills quantum mechanics are anyons: they obey exchange statistics $e^{ \pm i \pi / 2}= \pm i$. As we review in Section 3, the remaining three Hopf maps induce connections over the base space $S^{d-2}$ which subsequently arise as Berry connections in the quantum mechanics. Each is familiar to physicists: they are the $U(1)$ Dirac monopole, the $S U(2)$ Yang monopole [3], and the $S O(8)$ octonionic monopole [4, 5].

This quartet of Hopf bundles are the fab four of geometrical phases. The discrete $\mathbf{Z}_{2}$ holonomy was one of the earliest known Berry phases [6], while the Dirac monopole appeared as an example in Berry's original paper [7]. Both the Yang monopole and the octonionic monopole have also arisen in various contexts. The former was first introduced as a non-Abelian Berry phase in [8, 9], and has subsequently appeared in several condensed matter systems [10, 11, 12], with various properties explored in [13]. The $S O(8)$ octonionic monopole appeared previously in association with the eight-dimensional quantum Hall effect [14]. In this paper we will see how all these connections arise as (non-Abelian) Berry phases in Yang-Mills quantum mechanics with $N$ supercharges.

The present paper can be thought of as a follow-up to our recent work [15, 16] exploring the Berry phase that emerges in various supersymmetric quantum mechanics. In [15], we showed that certain $N=4$ quantum systems naturally give rise to the Dirac monopole and associated constructs such as the smooth 't Hooft-Polyakov monopole. In [16], we studied a quantum theory with $N=8$ supercharges and showed that a deformation of the Yang-monopole arises as the spin connection of a dual gravitational background. The Yang monopole and octonionic monopole also appeared in a related context in [17].

\section{D0-Brane Dynamics}

In this paper we study the $S U(2)$ super-Yang-Mills quantum mechanics with $N=2,4,8$ and 16 supercharges. The Lagrangians are the dimensional reduction of minimal super Yang-Mills in $d=3,4,8$ and 10 dimensions respectively, and each takes the form

$$
L=\frac{1}{2 g^{2}} \operatorname{Tr}\left(\left(\mathcal{D}_{0} X_{i}\right)^{2}+\sum_{i<j}\left[X_{i}, X_{j}\right]^{2}+i \bar{\psi} \mathcal{D}_{0} \psi+\bar{\psi} \Gamma^{i}\left[X_{i}, \psi\right]\right),
$$

where $X_{i}$ and $\psi_{\alpha}$ are both $s u(2)$ valued fields. The inverse coupling constant $1 / g^{2}$ is

the mass of the D0-brane. Lagrangians with different amounts of supersymmetry are 
distinguished by the number of scalar fields $X_{i}$, and the structure of the Grassmann parameters $\psi$. The scalar index runs over $i=1, \ldots, d-1$ and the Lagrangian describes the non-relativistic relative motion of a pair of D0-branes moving in $d-1=2,3,5$ and 9 spatial dimensions. The matrices $\Gamma^{i}$ satisfy the $d-1$ dimensional Euclidean Clifford algebra: $\left\{\Gamma^{i}, \Gamma^{j}\right\}=2 \delta^{i j}$. The spinors are real or complex, depending on the possible representations of the Clifford algebra:

- $N=2$ : For $d-1=2$, we may choose the real Pauli matrices $\Gamma^{1}=\sigma^{1}, \Gamma^{2}=\sigma^{3}$. The spinor $\psi_{\alpha}$, with $\alpha=1,2$, is also real.

- $N=4$ : For $d-1=3$, there are only complex representations of the Clifford algebra. We take the Pauli matrices $\Gamma^{i}=\sigma^{i}$. The spinor $\psi_{\alpha}$, with $\alpha=1,2$, is complex.

- $N=8$ : The Clifford algebra in $d-1=5$ dimensions is again complex. The complex spinor $\psi_{\alpha}$ has $\alpha=1, \ldots, 4$.

- $N=16$ : The $d-1=9$ Clifford algebra admits a real representation. The real spinor $\psi_{\alpha}$ has $\alpha=1, \ldots, 16$.

\subsection{The Born-Oppenheimer Approximation}

We work in the Born-Oppenheimer approximation, considering well-localized wavepackets describing D0-branes with separation $\hat{X}^{i}$. To this end, we expand around the classical background

$$
\left\langle X^{i}\right\rangle=\frac{1}{2} \hat{X}^{i} \sigma^{3}
$$

and study the effects of high-frequency modes of open strings stretched between the D0-branes. Our results are applicable in the weak coupling limit $\hat{X}^{3} \gg g^{2}$. This typically excludes the ground state of the full system, for which the wavefunction is localized close to the origin $\hat{X} \sim g^{2 / 3}$.

The background (3) breaks the gauge symmetry to the Cartan subalgebra: $S U(2) \rightarrow$ $U(1)$. The remnant $\mathbf{Z}_{2}$ Weyl group acts as $\hat{X}^{i} \rightarrow-\hat{X}^{i}$. The configuration space of the D0-branes is thus $\mathbf{R}^{d} / \mathbf{Z}_{2}$, reflecting the indistinguishability of the particles. We expand,

$$
X^{i}=\left\langle X^{i}\right\rangle+\frac{1}{2} x_{m}^{i} \sigma^{m}
$$

\footnotetext{
${ }^{1}$ More precisely, the theory with $N=16$ supercharges describes the dynamics of a pair of D0-branes in flat ten-dimensional spacetime. Lagrangians with less supersymmetry describe the dynamics of a pair of fractional D0-branes, trapped to lie at a suitable singularity in a $\mathrm{K} 3$, a CY 3 -fold, or a $G_{2}$ holonomy manifold.
} 
The bosonic commutator term in (2) provides oscillation frequencies for the fluctuations $x_{m}^{i}$. The fluctuations $x_{3}^{i}$, lying in the unbroken $U(1)$, are zero modes and describe the relative motion of the D0-brane pair in $\mathbb{R}^{d-1}$. In contrast, the $m=1,2$ components, lying in the broken part of the gauge group, pick up non-zero frequencies and describe the excitation of open strings stretched between the D0-branes. We form the complex combination

$$
z^{i}=x_{1}^{i}+i x_{2}^{i}, \quad i=1, \ldots, d-1
$$

which has charge +1 under the unbroken $U(1)$ gauge symmetry. Introducing the complex conjugate momentum $\pi_{i}$, the leading order free Hamiltonian for these off-diagonal bosonic modes is given by,

$$
H_{B}=\frac{1}{2 g^{2}}\left[\mathcal{P}_{i j}\left(\pi_{i} \bar{\pi}_{j}+\hat{X}^{2} z_{i} \bar{z}_{j}\right)+\ldots\right]
$$

where ... are interaction parts of the Hamiltonian which are perturbations of order $g / \hat{X}^{1 / 3}$. The Hamiltonian includes the projection operator

$$
\mathcal{P}_{i j}=\delta_{i j}-\frac{\hat{X}^{i} \hat{X}^{j}}{\hat{X}^{2}}
$$

and therefore describes only $d-2$ complex harmonic oscillators. These oscillators can be thought of as the transverse excitations of a string stretched between the D0-branes. The remaining degree of freedom falls victim to the broken gauge symmetry on the D0-branes; it is analogous to the scalar that is eaten by the Higgs mechanism in higher dimensions.

We may also expand the fermions around the background (3). Once again, the fermions $\left(\psi_{\alpha}\right)_{3}$, lying in the Cartan subalgebra, provide zero modes. Upon quantization, these fill out a $2^{N / 2}$ dimensional multiplet of states whose degeneracy is split only by interaction terms. In contrast, the off-diagonal components $\left(\psi_{\alpha}\right)_{1}$ and $\left(\psi_{\alpha}\right)_{2}$ have nonzero frequencies. Here it is convenient to differentiate between the $N=2,16$ cases, which have real fermions and the $N=4,8$ cases, which have complex fermions. In the former case, we introduce the complexified Grassmann parameter,

$$
\Psi=\psi_{1}+i \psi_{2}
$$

in terms of which the fermionic Hamiltonian is written as

$$
H_{F}^{N=2,16}=\frac{1}{2 g^{2}}\left[\Psi^{\dagger}(\hat{X} \cdot \Gamma) \Psi+\ldots\right]
$$


The $N=4,8$ cases have complex spinors from the outset. We may now form two linearly independent complex combinations

$$
\Psi=\frac{1}{\sqrt{2}}\left(\psi_{1}+i \psi_{2}\right) \quad, \quad \tilde{\Psi}=\frac{1}{\sqrt{2}}\left(\psi_{1}-i \psi_{2}\right)
$$

with respective charges +1 and -1 under the unbroken $U(1)$ gauge group. The fermionic part of the Hamiltonian is now given by

$$
H_{F}^{N=4,8}=\frac{1}{2 g^{2}}\left[\Psi^{\dagger}(\hat{X} \cdot \Gamma) \Psi-\tilde{\Psi}^{\dagger}(\hat{X} \cdot \Gamma) \tilde{\Psi}+\ldots\right]
$$

To summarize, the free part of the Hamiltonian $H=H_{B}+H_{F}$ for the massive oscillators contains $N / 2$ complex scalars and $N$ complex Grassmann parameters. The interaction Hamiltonian is of order $g / \hat{X}^{1 / 3}$.

\subsection{Quantization and the Hilbert Space}

In the Born-Oppenheimer approximation, we treat the massive oscillator states $z_{i}, \Psi_{\alpha}$ and $\tilde{\Psi}_{\alpha}$ quantum mechanically in the classical background $\hat{X}$. The zero frequency modes $\hat{X}^{i}$ and $\left(\psi_{\alpha}\right)_{3}$ are quantized subsequently. The approximation holds as long as $\hat{X} \gg g^{1 / 3}$ ensuring that we keep a separation of scales, meaning that the wavefunction for $\hat{X}$ should not have significant support near the origin where the two D0-branes approach. For example, this will be the case for excited states of orbiting D0-branes that carry large angular momentum. In the following section we will study the holonomy of the excited states of the massive oscillators as the two D0-branes orbit. We first briefly describe the Hilbert space of these states.

Working in the regime $\hat{X} \gg g^{1 / 3}$, we may restrict attention to the free theory. The $d-2$ massive complex scalars have ground state energy $E_{B}=(d-2) \hat{X} / 2 g^{2}$. One may construct the Hilbert space by acting with (suitably projected) creation operators $\mathcal{P}_{i j} a_{j}^{\dagger}$ and $\mathcal{P}_{i j} \bar{a}_{j}^{\dagger}$, where

$$
a_{i}^{\dagger}=\frac{\left(\hat{X} z_{i}-i \bar{\pi}_{i}\right)}{\sqrt{2 \hat{X}}} \quad, \quad \bar{a}_{i}^{\dagger}=\frac{\left(\hat{X} \bar{z}_{i}-i \pi_{i}\right)}{\sqrt{2 \hat{X}}}
$$

create quanta of charge +1 and -1 respectively under the unbroken $U(1)$ gauge symmetry. Canonical quantization for fermions gives the brackets $\left\{\Psi_{\alpha}, \Psi_{\beta}^{\dagger}\right\}=\delta_{\alpha \beta}$ and $\left\{\tilde{\Psi}_{\alpha}, \tilde{\Psi}_{\beta}^{\dagger}\right\}=\delta_{\alpha \beta}$. We build the fermionic Hilbert space $\mathcal{H}_{F}$ by picking a reference state $|0\rangle$, satisfying

$$
\Psi_{\alpha}|0\rangle=\tilde{\Psi}_{\alpha}|0\rangle=0
$$

We may then act upon $|0\rangle$ with $\Psi^{\dagger}$ in the $N=2,16$ theories, and $\Psi^{\dagger}$ and $\tilde{\Psi}^{\dagger}$ in the $N=4,8$ theories, to construct a Hilbert space of $\operatorname{dimension} \operatorname{dim}\left(\mathcal{H}_{F}\right)=2^{N}$. 
The free fermionic Hamiltonians (9) and (11) have a unique ground state $|\Omega\rangle$ with energy $E_{F}=-N \hat{X} / 4 g^{2}$. This ensures that the ground state energy of the full theory is $E_{0}=E_{B}+E_{F}=0$. The fermionic ground state always lies in the sector with half of the fermions excited. To describe it, we first introduce the fermionic projection operators

$$
P_{ \pm}=\frac{1}{2}\left(1 \pm \frac{\hat{X} \cdot \Gamma}{|\hat{X}|}\right)
$$

Then, for the $N=2,16$ theories, the (un-normalized) ground state is given schematically by,

$$
|\Omega\rangle=\left(P_{-} \Psi^{\dagger}\right)^{N / 2}|0\rangle
$$

while, for the $N=4,8$ theories, the (un-normalized) ground state is given by

$$
|\Omega\rangle=\left(P_{-} \Psi^{\dagger}\right)^{N / 4}\left(P_{+} \tilde{\Psi}^{\dagger}\right)^{N / 4}|0\rangle
$$

Note that $|\Omega\rangle$ is the ground state for the high frequency modes in the Born-Oppenheimer approximation. The question of whether a normalizable ground state of the full theory exists is more subtle, and irrelevant for our considerations. (It does for $N=16$, but is expected not to for $N=2,4,8)$.

The physical Hilbert space of the theory is subject to Gauss' law for the unbroken $U(1) \subset S U(2)$ gauge symmetry which ensures that all physical states are gauge neutral. The vacuum $|\Omega\rangle$ is assigned charge zero under the $U(1)$ and so survives Gauss' purge. Other states in the fermionic Hilbert space arise by acting on $|\Omega\rangle$ with combinations of the charge +1 states $P_{-} \Psi$ and $P_{-} \tilde{\Psi}^{\dagger}$ and the charge -1 states $P_{+} \Psi^{\dagger}$ and $P_{+} \tilde{\Psi}$. In each case, one may construct a state in the physical Hilbert space by dressing the fermionic state with appropriate powers of the charged bosonic operator $\mathcal{P}_{i j} a_{j}^{\dagger}$ and $\mathcal{P}_{i j} \bar{a}_{j}^{\dagger}$.

\section{Berry Phase and Hopf Maps}

In the previous section we constructed the Hilbert space over each background separation $\hat{X}^{i}$. In this section we are interested in how these Hilbert spaces evolve as the D0-branes orbit, and $\hat{X}^{i}$ traces a closed path $\Gamma$ in configuration space. The dynamical phase of a set of degenerate states $\left|\phi_{a}\right\rangle$ is accompanied by a (possibly non-Abelian) Berry holonomy, described by

$$
\left|\phi_{a}\right\rangle \longrightarrow P \exp \left(-i \oint_{\Gamma} A_{a b} \cdot \hat{X}\right)\left|\phi_{b}\right\rangle \quad \text { with } \quad\left(A_{i}\right)_{a b}=i\left\langle\phi_{b}\left|\frac{\partial}{\partial \hat{X}^{i}}\right| \phi_{a}\right\rangle
$$

We will see that for certain states in quantum mechanics with $N$ supercharges, the Berry connection $A$ is given by the associated Hopf map. We start by reviewing the the Hopf maps and connections in more detail. 
The Hopf map from $S^{N-1} \rightarrow S^{N / 2}$ is defined in the following manner. One starts with a commuting spinor $\chi_{\alpha}$, of the type described in Section 2: real for $N=2,16$ and complex for $N=4,8$. Imposing the normalization condition $\chi^{\dagger} \chi=1$ ensures that $\chi$ defines a point on $S^{N-1}$. The map to the base manifold $S^{N / 2}$ is then given by the bi-linear form

$$
n^{i}=\chi^{\dagger} \Gamma^{i} \chi
$$

where $\Gamma^{i}$ obey the $S O(d-1)$ Clifford algebra. One may check that $n^{i} n^{i}=1$ and hence $n^{i}$ defines a point on $S^{N / 2}$.

It is illustrative to stress the connection to the division algebras by reformulating the Hopf maps over the algebra $\mathbb{K}$ using the isomorphism (11). (For more details see, for example, [18]). The total manifold $S^{N-1}$ can be thought of as arising from the pair $\left(q_{1}, q_{2}\right) \in \mathbb{K}^{2}$, subject to the constraint $\left|q_{1}\right|^{2}+\left|q_{2}\right|^{2}=1$. From this pair we can define the vector $n^{i}, i=1 \ldots, d-1$,

$$
n^{i}=q^{\dagger} \Gamma^{i} q
$$

where $\Gamma^{i}$ still satisfy the $S O(d-1)$ Clifford algebra, hence justifying their name, but can now be thought of as basis elements of $\Gamma^{i} \in \operatorname{sl}(2 ; \mathbb{K})$, defined by

$$
\Gamma^{i}=\left(\begin{array}{cc}
0 & e_{i} \\
e_{i}^{\star} & 0
\end{array}\right) \quad i=1, \ldots, N / 2 \quad, \quad \Gamma^{d-1}=\left(\begin{array}{cc}
1 & 0 \\
0 & -1
\end{array}\right)
$$

where $e_{i}$ are the generators of the division algebra $\mathbb{K}$. The fact that these matrices obey the Clifford algebra reflects the isomorphism (11). One can check once again that $n^{i} n^{i}=1$, ensuring that this defines a map to the base manifold $S^{N / 2} \cong \mathbb{K P}^{1}$.

The zeroth Hopf map associated to $\mathbb{R}$ is the Möbius bundle $\mathbf{Z}_{2} \hookrightarrow S^{1} \rightarrow \mathbb{R P}^{1}$. We will shortly see how this arises in the theory with $N=2$ supercharges: the holonomy of the ground state is a minus sign whose role is to render the D0-branes anyonic. The remaining Hopf maps, associated to $\mathbb{C}, \mathbb{H}$ and $\mathbb{O}$, each define a connection $A$ over the base space $S^{N / 2}$, which describes how the base manifold is twisted inside the total space. These connections are given in terms of the projection operators $P_{ \pm}$defined in (14), where we identify $\hat{X}^{i} \equiv \hat{X} n^{i}$. Let $\lambda_{a}$ be the non-vanishing orthonormal eigenvectors of $P_{-}$: i.e. $P_{-} \lambda_{a}=\lambda_{a}$. The Hopf connection is defined by,

$$
A_{a b}=i \lambda_{b}^{\dagger} d \lambda_{a}
$$

For $N=4,8$ and 16, the maximum value of the index $a$ is $1,2,8$ and $A_{a b}$ is therefore a $U(1), U(2)$ and $S O(8)$ connection respectively. In the following, we will describe these connections in more detail and see how they arise as the Berry phase of certain states in the quantum mechanics. 


\section{1 $N=2$ and the Zeroth Hopf Map}

For the $N=2$ case, the ground state wavefunction undergoes a discrete holonomy as the D0-branes orbit. While the existence of this phase follows on general grounds from the degenerate nature of $H_{F}$ at the origin, it is instructive to review explicitly how it occurs.

The fermionic Hilbert space $\mathcal{H}_{F}$ consists of four states $|0\rangle, \Psi_{\alpha}^{\dagger}|0\rangle$ and $\Psi_{1}^{\dagger} \Psi_{2}^{\dagger}|0\rangle$. The ground state may be expanded as a linear combination of the middle sector,

$$
|\Omega\rangle=\lambda_{\alpha} \Psi_{\alpha}^{\dagger}|0\rangle
$$

with $\lambda_{\alpha}$ the negative eigenvector of $\hat{X} \cdot \Gamma$. We choose to work with the real basis of gamma matrices $\Gamma^{1}=\sigma^{1}$ and $\Gamma^{2}=\sigma^{3}$ and introduce polar coordinates

$$
X^{1}=X \sin \theta \quad, \quad X^{2}=X \cos \theta
$$

Then the groundstate eigenvector is

$$
\vec{\lambda}=\frac{1}{\sqrt{2+2 \cos \theta}}\left(\begin{array}{c}
-\sin \theta \\
1+\cos \theta
\end{array}\right)
$$

where we have resolved the square-root sign ambiguity in favour of the positive. The evolution of the eigenvector as $\theta$ is adiabatically varied is shown in the figure. We see that as $\theta$ varies from 0 to $2 \pi, \vec{\lambda}$ returns to $-\vec{\lambda}$. This is the manifestation of the zeroth Hopf map: $S^{1} \rightarrow S^{1}$.

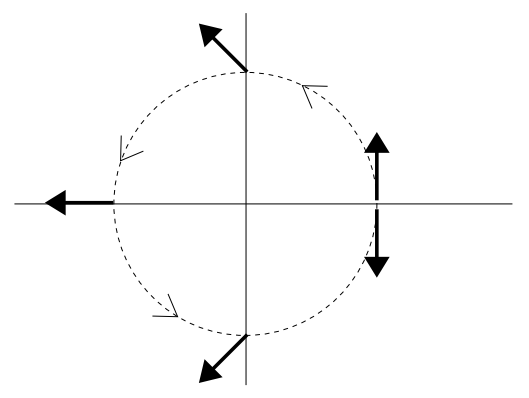

Figure 1: The $\mathbf{Z}_{2}$ holonomy as the eigenvector encircles the degeneracy at the origin.

This $\mathbf{Z}_{2}$ Berry phase is inherited by the ground state of the D0-branes. After a single orbit, the wavefunction returns to $|\Omega\rangle \mapsto-|\Omega\rangle$. Yet such an orbit is equivalent to two exchanges of the D0-branes. Since the branes are indistinguishable particles, upon a single exchange, the D0-brane wavefunction picks up the phase $e^{ \pm i \pi / 2}= \pm i$, where the \pm sign depends on whether the exchange proceeds clockwise or anti-clockwise. We learn that the D0-branes are anyons [19, 20]. 
It is worthwhile re-deriving the fact that the D0-branes are anyons by studying a single exchange, rather than a complete orbit. Let us start with the D0-branes separated by $\hat{X}^{i}=(0, \hat{X})$, for which the ground state is given by $|\Omega\rangle_{+}=\Psi_{2}^{\dagger}|0\rangle$. After an anti-clockwise rotation to $\hat{X}^{i}=(0,-\hat{X})$, we see from (24) that the ground state adiabatically evolves into $|\Omega\rangle_{-}=-\Psi_{1}^{\dagger}|0\rangle$. The $\mathbf{Z}_{2}$ Weyl symmetry ensures that the Hilbert space constructed over $\hat{X}^{i}$ is physically identified with the Hilbert space over $-\hat{X}^{i}$. Thus in order to understand the phase picked up by the ground state, we need to understand the map between the Hilbert spaces constructed over $\pm \hat{X}^{i}$ induced by the Weyl group.

The action of the $\mathbf{Z}_{2}$ Weyl group is given by,

$$
\mathbf{Z}_{2}: \quad \hat{X}^{i} \mapsto-\hat{X}^{i} \quad ; \quad z^{i} \mapsto-z^{\dagger i} \quad ; \quad \Psi \mapsto-\Psi^{\dagger}
$$

From the latter of these actions, we see that the reference state $|0\rangle_{+}$defined by (13) at $\hat{X}^{i}$ is mapped to $\Psi_{1}^{\dagger} \Psi_{2}^{\dagger}|0\rangle_{-}$at $-\hat{X}^{i}$, up to a phase $\omega$,

$$
\mathbf{Z}_{2}: \quad|0\rangle_{+} \mapsto \omega \Psi_{1}^{\dagger} \Psi_{2}^{\dagger}|0\rangle_{-}
$$

The question is: what is $\omega$ ? We can answer this by following the fate of the state $\Psi_{1}^{\dagger}|0\rangle_{+}$under two actions of the Weyl group,

$$
\Psi_{1}^{\dagger}|0\rangle_{+} \mapsto-\omega \Psi_{2}^{\dagger}|0\rangle_{-} \mapsto-\omega^{2} \Psi_{1}^{\dagger}|0\rangle_{+}
$$

Insisting that the Weyl group action squares to the unit operator, we learn that $\omega= \pm i$. We now use this knowledge to map the state $|\Omega\rangle_{-}$above back to an element of the Hilbert space over $\hat{X}^{i}=(0, \hat{X})$. We see,

$$
|\Omega\rangle_{+} \stackrel{\text { adiabatic }}{\longrightarrow}|\Omega\rangle_{-} \stackrel{\mathbf{z}_{2}}{\longrightarrow} \mp i|\Omega\rangle_{+}
$$

The upshot is that the ground state of the two D0-branes indeed picks up the phase factor $\pm i$ upon exchange.

Note that we have still to quantize the $U(1) \subset S U(2)$ Cartan subalgebra. In particular, this includes the massless fermions $\left(\psi_{3}\right)_{\alpha}$, which double the Hilbert space. The resulting states have conjugate exchange statistics. If one state picks up a phase $+i$ upon an anti-clockwise rotation, the other state picks up a phase $-i$.

\section{2 $N=4$ and the First Hopf Map}

We now turn to D0-branes moving in $d-1=3$ spatial dimensions. As explained in Section 2, the Hilbert space is constructed by acting with bosonic and fermionic creation 
operators on the reference state $|0\rangle$. Each of these creation operators is accompanied by a projection operator: $\mathcal{P}$ for bosons, defined in (7), and $P_{ \pm}$for fermions, defined in (14). These two types of projection operators give rise to two types of Berry holonomy:

The projection operators $\mathcal{P}$ restrict the bosonic excitations to lie tangent to the sphere $S^{2}$ at fixed $|\hat{X}|$. As we saw above, this can be traced to the implementation of the $S U(2)$ gauge symmetry and, from the string theory perspective, is the familiar statement that stretched strings have only transverse excitations. The net result is that as the D0-branes orbit, excited states that include bosonic excitations undergo a Berry holonomy as tangent vectors on the sphere $S^{2}$. This is the same kind of non-Abelian holonomy that was described in the original paper of Wilczek and Zee [21].

Fermionic excitations are accompanied by the projection operators $P_{ \pm}$(14). To be concrete, let's focus on the operator $P_{-}$. We introduce the normalized eigenvector $P_{-} \lambda=\lambda$. Acting with the creation operator $P_{-} \Psi^{\dagger}$ means creating the normalized state $|\lambda\rangle=\lambda_{\alpha} \Psi_{\alpha}^{\dagger}|0\rangle$. As $\hat{X}^{i}$ varies adiabatically, this state undergoes a holonomy described by the Berry connection,

$$
A_{i}=i\left\langle\lambda\left|\frac{\partial}{\partial \hat{X}^{i}}\right| \lambda\right\rangle
$$

which is the connection of the Hopf map (21). An explicit form of the connection requires a choice of gauge which, in this context, means a chosen phase for the eigenvector $\lambda$. We choose $\lambda_{1} \in \mathbb{R}$, a choice which is valid everywhere except along the positive $\hat{X}^{3}$ axis. In this gauge, the Berry connection takes the familiar form of the Dirac monopole,

$$
A_{i}=\frac{-\hat{X}^{j}}{2 \hat{X}\left(\hat{X}-\hat{X}^{3}\right)} \epsilon_{i j} \quad i=1,2 \quad, \quad A_{3}=0
$$

The Hopf-Berry connection has first Chern class -1 over the sphere $S^{2}$, meaning that the integral of the field strength $F=d A$ yields,

$$
c_{1}=\frac{1}{2 \pi} \int_{S^{2}} F=-1
$$

Acting with the operator $P_{+} \Psi^{\dagger}$ results is a state whose Berry connection has Chern class +1 . The ground state in the $N=4$ theory is given by

$$
|\Omega\rangle=N^{\prime}\left(P_{-} \Psi^{\dagger}\right)\left(P_{+} \tilde{\Psi}^{\dagger}\right)|0\rangle \equiv\left(\lambda_{\alpha} \Psi_{\alpha}^{\dagger}\right)\left(\tilde{\lambda}_{\beta} \tilde{\Psi}_{\beta}^{\dagger}\right)|0\rangle
$$

where $N^{\prime}$ is a normalization factor, while $\lambda(\tilde{\lambda})$ is the normalized non-zero eigenvector of $P_{\mp}$. The presence of the two, opposite, projection operators ensures that the ground state does not pick up a Berry phase as the D0-branes orbit. 
Excited states do pick up a Berry phase, given by the sum of the phases associated to the relevant projection operators. For example, a degenerate pair of states obeying Gauss' law is given by

$$
\left|\phi_{-}\right\rangle=\left(\lambda_{\alpha} \Psi_{\alpha}^{\dagger}\right)\left(\lambda_{\alpha} \tilde{\Psi}_{\alpha}^{\dagger}\right)|0\rangle \quad, \quad\left|\phi_{+}\right\rangle=\left(\tilde{\lambda}_{\alpha} \Psi_{\alpha}^{\dagger}\right)\left(\tilde{\lambda}_{\alpha} \tilde{\Psi}_{\alpha}^{\dagger}\right)|0\rangle
$$

These states have energy $E=E_{B}+E_{F}=\hat{X} / g^{2}$ and describe two strings attached to the D0-branes. (They are part of a triplet of excited spin 1 states). From the discussion above, we see that these states pick up a Berry phase arising from a magnetic monopole of charge $q= \pm 2$. Physically, this means that the D0-branes orbit as charged particles as if in the presence of a magnetic monopole fixed at their centre. In a semi-classical analysis, the orbits are no longer restricted to lie on a plane, but rather lie on a cone with opening angle $\cos \theta=-q /(2 J+q)$, where $J$ is angular momentum of the spinning D0-branes. The energy $E$ these rotating states scales as $E^{3} \sim g^{2} J(J+q)$.

\section{3 $N=8$ and the Quaternionic Hopf Map}

The discussion for the $N=8$ theory is very similar to the $N=4$ theory above. The unique ground state of the system (16) does not undergo a Berry phase. Excited states do. The typical state undergoes a non-Abelian holonomy arising from the sum of bosonic and fermionic Berry connections. Once again, bosonic excitations give rise to a holonomy in which states transform as tangent vectors on $S^{5}$. More interesting for the present discussion are the fermionic excitations. The projection operator $P_{-}$ now has a pair of orthonormal eigenvectors: $P_{-} \lambda_{a}=\lambda_{a}, a=1,2$. This means that the resulting Berry holonomy of the states $\left|\lambda_{a}\right\rangle=\lambda_{a \alpha} \Psi_{\alpha}^{\dagger}|0\rangle$ is described by a $U(2)$ connection,

$$
\left(A_{i}\right)_{a b}=i\left\langle\lambda_{a}\left|\frac{\partial}{\partial \hat{X}^{i}}\right| \lambda_{b}\right\rangle
$$

Explicit computation shows that this is actually an $S U(2)$ connection, known as the Yang-monopole [3]. A suitable choice of gamma matrices and gauge, can be found in [16. The resulting connection is,

$$
\left(A_{i}\right)_{a b}=\frac{-\hat{X}^{j}}{2 \hat{X}\left(\hat{X}-\hat{X}^{5}\right)} \eta_{i j}^{m} \sigma_{a b}^{m} \quad i=1,2,3,4 \quad, \quad A_{5}=0
$$

where $\eta_{\mu \nu}^{m}$ are the self-dual $4 \times 4$ 't Hooft matrices and $\sigma_{a b}$ are the Pauli matrices. The Yang monopole is perhaps more familiar when viewed as a connection restricted to $S^{4}$, where it is simply the $S O(5)$ invariant instanton satisfying $F=-{ }^{\star} F$ with second Chern class,

$$
c_{2}=\frac{1}{8 \pi^{2}} \int_{S^{4}} \operatorname{tr}(F \wedge F)=-1
$$


where the non-Abelian field strength is defined by $F_{i j}=\partial_{i} A_{j}-\partial_{j} A_{i}-i\left[A_{i}, A_{j}\right]$. States constructed from the projection operators $P_{+}$undergo a Berry holonomy associated with a Yang-monopole of Chern class $c_{2}=+1$.

\section{$3.4 N=16$ and the Last Hopf Map}

Details for the theory with $N=16$ supercharges are again similar to those above: the vacuum (15) does not pick up a Berry connection, while bosonic excitations transform as tangent vectors on $S^{8}$. The fermionic excitations are associated to projection operators $P_{ \pm}$, which are now $16 \times 16$ real matrices. We once again define the 8 orthonormal, real eigenvectors $P_{-} \lambda_{a}=\lambda_{a}$, with $a=1, \ldots, 8$. States in the quantum mechanics involving fermions undergo a holonomy arising from the $S O(8)$ Berry connection,

$$
\left(A_{i}\right)_{a b}=i\left\langle\lambda_{b}\left|\frac{\partial}{\partial \hat{X}^{i}}\right| \lambda_{a}\right\rangle
$$

with $\left|\lambda_{a}\right\rangle=\lambda_{a \alpha} \Psi_{\alpha}^{\dagger}|0\rangle$. We refer to the resulting connection as the $S O(8)$ octonionic

monopole. It was constructed in [4, 5]. A simple expression for the connection can be found in [14]

$$
\left(A_{i}\right)_{a b}=\frac{-\hat{X}^{j}}{2 \hat{X}\left(\hat{X}-\hat{X}^{9}\right)} \Sigma_{i j} \quad i=1, \ldots, 8 \quad, \quad A_{9}=0
$$

where $\Sigma_{i j}$ are the 28 generators of $S O(8)$ Lie algebra, defined in terms of the $\Gamma$ matrices. An explicit form can be given if we choose a suitable representation for the generators of the octonions $e_{i}, i=1 \ldots 8$ in (20), in terms of $8 \times 8$ real matrices. We choose the unit matrix to correspond to $e_{8}=1$. Then $\Sigma_{i j}=-\frac{1}{2}\left[e_{i}, e_{j}\right]$, for $i=1, \ldots, 7$ and $\Sigma_{i 8}=e_{i}$ [14]. The non-Abelian field strength restricted to $S^{8}$ satisfies the generalized self-duality condition $F \wedge F=-{ }^{\star} F \wedge F$, with

$$
\frac{1}{4 !(2 \pi)^{4}} \int_{S^{8}} \operatorname{tr}(F \wedge F \wedge F \wedge F)=-1
$$

As in previous cases, states associated to $P_{+}$undergo a Berry holonomy with $c_{4}=1$.

It is intriguing to see the octonionic monopole appearing in the Matrix theory for D0branes in this fashion, although its physical significance in M-theory remains obscure. Nonetheless, it is tempting to speculate. Firstly, recall that there is a precedent for the appearance of Berry's phase in Matrix theory: the membrane feels the magnetic field of the five-brane through the appearance of a Berry connection [22]. In this case, the abstract Berry connection is recast as a physical magnetic field. It would be very interesting if a similar M-theory interpretation could be given in the present case. We note in passing that a physical octonionic monopole is conjectured to act as the end-point for an open heterotic string [23]. 


\section{Acknowledgments}

We would like to thank Freddy Cachazo, Joe Polchinski, Savdeep Sethi and Paul Townsend for useful discussions. J.S. gratefully acknowledges the University of Chicago, Harvard and the Perimeter Institute for hospitality and travel support while this paper was being written up. D.T. thanks the Isaac Newton Institute for their kind hospitality while this work was undertaken. C.P. is supported by an EPSRC studentship. J.S. is supported by a research fellowship from Trinity College, Cambridge. D.T. is supported by the Royal Society.

\section{References}

[1] T. Kugo and P. K. Townsend, Supersymmetry And The Division Algebras, Nucl. Phys. B 221, 357 (1983).

[2] J. M. Evans, "Supersymmetric Yang-Mills Theories and Division Algebras", Nucl. Phys. B 298, 92 (1988).

[3] C. N. Yang, "Generalization Of Dirac's Monopole To SU(2) Gauge Fields," J. Math. Phys. 19, 320 (1978).

[4] B. Grossman, T. W. Kephart and J. D. Stasheff, "Solutions To Yang-Mills Field Equations In Eight-Dimensions And The Last Hopf Map," Commun. Math. Phys. 96, 431 (1984) [Erratum-ibid. 100, 311 (1985)].

[5] D. H. Tchrakian, "Spherically Symmetric Gauge Field Configurations With Finite Action In 4 P-Dimensions ( $P=$ Integer)," Phys. Lett. B 150, 360 (1985).

[6] G. Herzberg and H. C. Longuet-Higgins, "Intersection of potential energy surfaces in polyatomic molecules", Discuss. Faraday Soc., 1963, 35, 77

[7] M. V. Berry, "Quantal phase factors accompanying adiabatic changes," Proc. Roy. Soc. Lond. A 392, 45 (1984).

[8] J. E. Avron, L. Sadun, J. Segert and B. Simon, "Topological Invariants in Fermi Systems with Time-Reversal Invariance", Phys. Rev. Lett. 61, 1329 (1988); "Chern Numbers, Quaternions and Berry's Phases in Fermi Systems", Commun. Math. Phys. 124 (1989) 595.

[9] M. G. Benedict, L. G. Feher and Z. Horvath, "Monopoles and Instantons from Berry's Phase," J. Math. Phys. 30, 1727 (1989).

[10] E. Demler and S-C. Zhang, "Non-Abelian holonomy of BCS and SDW quasiparticles," Annals Phys. 271 (1999) 83 arXiv:cond-mat/9805404.

[11] S. Murakami, N. Nagaosa, S-C. Zhang, "SU(2) Non-Abelian Holonomy and Dissipationless Spin Current in Semiconductors", Phys. Rev. B69, 235206 (2004), arXiv:cond-mat/0310005 v3] 
[12] C-H. Chern, H-D. Chen, C. Wu, J-P. Hu, S-C. Zhang, "Non-abelian Berry's phase and Chern numbers in higher spin pairing condensates", Phys. Rev. B69, 214512 (2004) arXiv:cond-mat/0310089v2].

[13] P. Lévay, Geometrical description of SU(2) Berry phases, Phys. Rev. A 411990 2837; "Quaternionic Gauge Fields And The Geometric Phase," J. Math. Phys. 32, 2347 (1991); "NonAbelian Born-Oppenheimer electric gauge force and the natural metric on Hilbert subspaces," Phys. Rev. A 45, 1339 (1992);

[14] B. A. Bernevig, J. p. Hu, N. Toumbas and S. C. Zhang, "The Eight Dimensional Quantum Hall Effect and the Octonions," Phys. Rev. Lett. 91, 236803 (2003) arXiv:cond-mat/0306045.

[15] C. Pedder, J. Sonner and D. Tong, "The Geometric Phase in Supersymmetric Quantum Mechanics," arXiv:0709.0731 [hep-th].

[16] C. Pedder, J. Sonner and D. Tong, "The Geometric Phase and Gravitational Precession of D-Branes," arXiv:0709.2136 [hep-th].

[17] B. Chen, H. Itoyama and H. Kihara, "Nonabelian Berry phase, Yang-Mills instanton and USp(2k) matrix model," Mod. Phys. Lett. A 14, 869 (1999) arXiv:hep-th/9810237; "Nonabelian monopoles from matrices: Seeds of the spacetime structure," Nucl. Phys. B 577, 23 (2000) arXiv:hep-th/9909075].

[18] J. C. Baez, "The Octonions," arXiv:math/0105155.

[19] J. M. Leinaas and J. Myrheim, "On the theory of identical particles," Nuovo Cim. B 37 (1977) 1 .

[20] F. Wilczek, “Quantum Mechanics Of Fractional Spin Particles,” Phys. Rev. Lett. 49, 957 (1982).

[21] F. Wilczek and A. Zee, "Appearance Of Gauge Structure In Simple Dynamical Systems," Phys. Rev. Lett. 52, 2111 (1984).

[22] M. Berkooz and M. R. Douglas, "Five-branes in M(atrix) theory," Phys. Lett. B 395, 196 (1997) arXiv:hep-th/9610236.

[23] J. Polchinski, “Open heterotic strings," JHEP 0609, 082 (2006) arXiv:hep-th/0510033. 